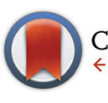

CrossMark

$\leftarrow$ click for updates

Cite this: Food Funct., 2016, 7, 464

\section{Glycemic index and microstructure analysis of a newly developed fiber enriched cookie $\uparrow$}

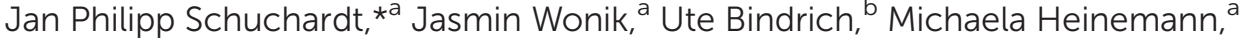 \\ Heike Kohrs, ${ }^{a}$ Inga Schneider, ${ }^{a}$ Katharina Möller ${ }^{a}$ and Andreas Hahn ${ }^{a}$
}

Received 17th September 2015 Accepted 16th October 2015

DOI: 10.1039/c5fo01137j www.rsc.org/foodfunction

\begin{abstract}
A diet with a high glycemic index (GI) is associated with an elevated risk for obesity or type 2 diabetes. We investigated the Gl of a newly-developed fiber enriched cookie and characterized the microstructure of ingredients used. In a study with 26 non-diabetic healthy volunteers it was shown that the fiber enriched cookie has a Gl of 58.9 in relation to white bread as reference. Using a conversion factor of 1.4, the Gl of the fiber enriched cookie in relation to a glucose-solution is 42.0 and can be classified as a low-Gl food. Postprandial insulin concentration was significantly lower after consumption of fiber enriched cookies compared to white bread. Glucose release after in vitro digestion was significantly lower from fiber enriched cookies compared to other cookies tested. In addition to its high percentage of fiber, the cookies' low Gl can be attributed to the limited gelatinization potential of the starch granules found in the ingredients used. Using confocal laser scanning microscopy it is shown that starch granule surface area of whole grain barley flour, spelt flour and oat flakes bears cluster-shaped protein-NSPS complexes that preferentially absorb water in conditions of water shortage and thereby prevent starch gelatinization.
\end{abstract}

\section{Introduction}

The glycemic index (GI) indicates the extent to which a carbohydrate containing food affects postprandial blood glucose levels compared with a reference product (glucose or white bread) containing the same amount of available carbohydrate. Foods with a high GI cause a high increase in postprandial blood glucose concentrations, while the increase is less pronounced for foods with a low GI. A high postprandial blood glucose response, in turn, triggers a disproportionately high insulin response and is associated with the development of hyperinsulinemia and insulin resistance. A diet of foods that has a high GI is associated with obesity, ${ }^{1}$ type 2 diabetes mellitus (DM2), ${ }^{2}$ coronary heart disease ${ }^{3}$ and colon cancer. ${ }^{4} \mathrm{~A}$ reduction in glycemic load is associated with reduced body weight. ${ }^{5}$ Therefore, the concept of eating foods with a low GI in the dietary therapy of DM2 and obesity is included in various international guidelines. ${ }^{6,7}$

For starch-containing foods like baked goods, the GI largely depends on the starch granules' accessibility to starch-splitting

${ }^{a}$ Institute of Food Science and Human Nutrition, Leibniz University of Hannover, Germany. E-mail: Schuchardt@nutrition.uni-hannover.de; Fax: +49 (0)511 762 5729; Tel: +49 (o)511 7622987

${ }^{b}$ Center of Food Physics, German Institute of Food Technologies, Quakenbrück, Germany

$\dagger$ Electronic supplementary information (ESI) available. See DOI: 10.1039/ c5fo01137j enzymes. Native starch exists in the form of compact starch granules that are made up of amylose and amylopectin. Starch can change its structure and gelatinize when water is present, due to thermal effects or because of shear forces. Native starch granules are only vulnerable to enzymes in the surface layers, while gelatinization changes the structure, enlarges the surface area and thereby increases the starch's vulnerability to enzymes. ${ }^{8,9}$ This increases the starch's digestibility and thus also the GI. According to own findings (un-published so far), in addition to protein adsorption, non-starch polysaccharides (NSPS) such as glucans and pentosans also bind to starch granules' surface layers; these lower the starch granules' vulnerability to enzymes. Soluble fibers like NSPS thus play a significant role in reducing GI. They not only reduce starch gelatinization in the food itself but also, because of their gelforming characteristics, slow down glucose absorption in the small intestine. ${ }^{10,11}$

The present study determined the GI of a newly developed fiber enriched cookie and described the microstructural and techno-functional characteristics of the ingredients used. The cookie's glucose and insulin responses were investigated in a nutritional physiological study with non-diabetic volunteers. Additionally, in vitro digestion of the fiber enriched cookies was carried out to compare the glucose release with short bread and digestive cookies. The gelatinization characteristics of the starch-containing raw materials and the microstructure of the starches they contain were analytically and microscopically investigated. 


\section{Materials and methods}

To determine the blood glucose and insulin effects of the fiber enriched cookies, a nutritional physiological study was conducted at the Institute of Food Sciences and Human Nutrition at Leibniz University of Hannover. The tests on starch gelatinization characteristics and microstructure were conducted at the German Institute of Food Technologies in Quakenbrück, Germany.

\subsection{Test products}

The fiber enriched cookie investigated in the study was developed and manufactured by Bahlsen GmbH \& Co. KG (Hannover, Germany). The ingredients were (in descending order): Flour (whole grain wheat flour, wheat flour, spelt flour), sucrose, oat flakes, sunflower oil, hazelnuts, oligo fructose, cocoa, baking agent: ammonium and sodium carbonate, oat fiber, whole grain barley flour, salt, flavour, skimmed milk powder, emulsifier: lecithin, acidifier: citric acid, cocoa butter. Sensory analysis according to the sequential monadic approach showed that the fiber enriched cookies reached the action standard for overall liking and therefore shows a significant acceptance level (data not shown). For compliance reasons, GI was not determined by means of the standard method of the Food and Agriculture Organization of the United Nations (FAO) using a glucose solution. ${ }^{12}$ Instead, subjects were given conventional white bread as a reference product to determine the GI. The nutrient content of the test and reference products used were tested in the LUFA ITL GmbH and biotask AG analytic laboratories. Total glucan and pentosan analysis in the test and reference products as well as the polysaccharide component analysis in starchy raw materials were carried out in the German Institute of Food Technologies.

\subsection{Degree of gelatinization of test products}

Since starch gelatinization is an endothermic process under the conditions of available water and atmospheric pressure, heat supply is necessary to induce this reaction. Therefore, the degree of gelatinization of the test and reference products, as well as a butter cookie (provided by Bahlsen GmbH \& Co KG) for comparison, were determined using differential scanning calorimetry (DSC). The foods to be tested were heated with an excess of water (starch/water ratio: 1/4 w/w) in the DSC measuring cell and the integral transition enthalpy. Because proteins in baked goods are already irreversibly denatured and other components (e.g., lipids) do not contribute to the endothermic reaction in the temperature range of starch gelatinization, the transition enthalpy is closely related to the starch's gelatinization enthalpy. The degree of gelatinization was calculated related to analytically determined total starch content of the products.

\subsection{Gelatinization potential of separated starch from starch- containing raw materials}

To determine the gelatinization potential of starch from soft wheat dunst and whole grain wheat flour, the native starch was first separated out through elutriation. For the other starchcontaining raw materials (whole grain barley flour, spelt flour, oat flakes), separation was performed by suspending the ground products in water, followed by separation using centrifugation, as the starch granules have the greatest density compared with the other components. The starch obtained in this process was subsequently dried non-thermally using ethanol. For measurement of the enthalpy, the native or partially gelatinized starch was weighed into standard DSC aluminium pans and heated under following conditions: sample mass about $50 \mathrm{mg}$ (10 mg starch $+40 \mathrm{mg}$ water); starting temperature $10{ }^{\circ} \mathrm{C}$; final temperature $98{ }^{\circ} \mathrm{C}$; ramp rate $2 \mathrm{~K} \mathrm{~min}^{-1}$. The resulting heat flow from the endothermic reaction was measured and the enthalpy was calculated as surface area between heat flow function and baseline (in reference to the initial starch weight). To measure the residual enthalpy after partial gelatinization of the starch from the raw materials, gelatinization was carried out with only $40 \%$ water (based on the dry mass (DM) starch), i.e., under conditions of water deprivation. The gelatinization state of these samples was then tested using light microscopy using potassium iodide solution (Lugol's solution) for labeling of starch. In a second step, the residual enthalpy was tested using DSC under the condition of water excess (see above).

\subsection{Determination of protein and starch in starch-containing raw materials}

The raw protein content was determined via the nitrogen content by the Kjeldahl method using the official ASU 18.00-5 method according to $\S 64$ of the German food and feed code (LFGB). The starch content was determined polarimetrically using the official ASU F13.00 method according to § 64 LFGB.

\subsection{Determination of glucan and pentosan attachments to separated starch granules}

The analysis of the $\beta$-Glucan content was performed photometrically according to McCleary and Codd $(1991)^{13}$ with the commercially available Enzyme Kit K-BGLU 07/11 from Megazyme (Wicklow, Ireland). The determination of pentosan content was performed with the phloroglucinol method according to Douglas 1981 based on the optimized version by Kiszonas et al. (2012). ${ }^{14}$

\subsection{Topography and adhesiveness of the surface area of separated starch granules}

The topography and adhesiveness of the surface area of the starch granules was characterized using atomic force microscopy (AFM). Therefore, starch granules were glued onto a mica disc using two-step-preparation method according to Neuner (G3305A, Plano GmbH, Wetzlar, Germany). AFM experiments were carried out using a MFP-3D SA (Asylum Research, Santa Barbara, California, U.S.A.) with standard silicon cantilevers of a nominal spring constant of $2 \mathrm{~N} \mathrm{~m}^{-1}$ (AC 240, Olympus Corporation, Tokyo, Japan). Topographical imaging was performed in intermittent contact mode with a cantilever resonant frequency around $75 \mathrm{kHz}$. Adhesion forces 
were calculated after performing $40 \times 40$ force-versus-distancecurves per force map of a size of $2 \times 2 \mu \mathrm{m}$ in contact mode. For each tip, exact cantilever's spring constant was determined via thermal noise method. ${ }^{15}$ Contact trigger point was $1 \mathrm{~V}$; scan velocity was $2 \mu \mathrm{m} \mathrm{s}^{-1}$. Two force maps per granule at different surface areas were examined, maps were captured as duplicates. Per sample, at least 8 granules were analyzed. Force maps were converted into a histogram in which quantity of occurrence of a certain adhesion force per map in \% was opposed to each other. Images and maps were measured and analyzed using IGOR Pro Software (WaveMetrics, Portland, USA) and Microsoft Excel 2010.

\subsection{Detecting protein-NSPS complexes on separated starch granules}

Confocal laser scanning microscopy (CLSM) Nikon ECLIIPSE E 600 (Nikon, Düsseldorf, Germany) with rhodamine R (reacting with amine groups of molecules) as fluorescent dye (absorption $570 \mathrm{~nm}$; emission $590 \mathrm{~nm}$, effecting red color in the micrographs) was performed in order to visualize proteins attached to the starch granules. Complexes of proteins and NSPS can be identified as regions of highly intensive coloring. This is caused by strong interactions between proteins and swollen NSPS molecules resulting in a compact structure. Separated starch granules were suspended in glycerine and mixed with the fluorescing dye. Adsorption of the dye at protein molecules is diffusion controlled and takes about 2 hours. Laser beam is focused on an appropriate image plane. CLSM micrographs are saved electronically.

\section{$2.8 \quad$ In vitro digestion assays}

For each sample $200 \mathrm{~g}$ cookies (short bread, digestive cookies [commercial products] and fiber enriched cookies) and white bread were finely crushed in a kitchen shredder (Moulinette, Krups, Offenbach/Main, Germany) and mixed. $100 \mathrm{mg}$ sample was suspended in $25 \mathrm{ml}$ phosphate buffer (ph 6.9) and homogenized with an Ultra-turrax ${ }^{\circledR}$ (IKA®, Staufen, Germany) for 30 s. Subsequently, 10U invertase (Sigma-Aldrich, Seelze, Germany), 25U pancreatin (Merck, Darmstadt, Germany) and 40U amyloglucosidase (Sigma-Aldrich, Seelze, Germany) were added. Samples were gently shacken and put in a water bath at $37{ }^{\circ} \mathrm{C}$. At each time point after $0,15,30,60,120$, and $180 \mathrm{~min}$ prior incubation $1 \mathrm{ml}$ was taken from the samples and transferred into a preheated tube. To stop enzymatic reaction samples were incubated for $5 \mathrm{~min}$ at $99^{\circ} \mathrm{C}$ and $750 \mathrm{rpm}$ in a thermomixer (Eppendorf, Hamburg, Germany). Samples were stored in a fridge until spectrometric measurement. Directly before spectrometric measurement $4 \mathrm{ml}$ de-ionized water was added to each sample and centrifuged for $5 \mathrm{~min}$ at $2000 \mathrm{rpm}$. Glucose concentrations were measured using a D-Glucose testkit (R-Biopharm, Darmstadt, Germany) according to the manufactures protocol and using a spectral photometer (Specord 205, Analytik Jena, Jena, Germany). Experiments were repeated eightfold.

\subsection{Study design of the nutritional physiological study}

The nutritional physiological study was designed in accordance with the Helsinki Declaration and conducted in accordance with Good Clinical Practice (GCP) principles and received a positive ethics vote. The study consisted of a screening and an intervention phase and lasted four weeks. During the intervention, four tests took place at intervals of 6 to 8 days. On test days, fasting blood samples were taken to determine the zero value (glucose and insulin); participants' body weight and height were also measured. Participants subsequently received the test product to be investigated and the reference product. Each portion of the test product $(84.5 \mathrm{~g}, 402 \mathrm{kcal})$ or the reference product $(112 \mathrm{~g}, 272 \mathrm{kcal})$ was equivalent to a $50 \mathrm{~g}$ amount of available carbohydrates. The participants were instructed to thoroughly chew and consume the test and reference along with $300 \mathrm{ml}$ water within a maximum of five minutes. The twoarmed study was conducted in a cross-over design, with repeat determination of the test and reference product, in which each subject received both the cookies and the reference product two times. During the intervention phase, the subjects were given $250 \mathrm{ml}$ of water after 60 and 120 minutes. To determine blood glucose and insulin concentrations, blood was drawn seven times (basal, 15, 30, 45, 60, 90 and 120 min after intake of the test or reference products). The timing of the subsequent blood samples was determined on the basis of the first bite taken during consumption of the test meals. The blood samples were prepared and analyzed by the Hannover Medical Care Center of the LADR network. The blood glucose concentration was measured in $2.4 \mathrm{ml}$ of venous sodium fluoride whole blood. The insulin concentration was measured in $2.7 \mathrm{ml}$ of serum. After each sampling, the blood samples were immediately cooled to $4-6^{\circ} \mathrm{C}$ in order to prevent breakdown of the blood glucose in the monovette. Any adverse events were recorded in order to monitor the tolerability of the cookies.

\subsection{Subjects}

Healthy, non-smoking, non-diabetic men and women $n=26$ (male: 14, female: 12) were included in the nutritional physiological study. Subjects were recruited via notices posted at Leibniz University of Hannover. The inclusion criteria for participation were a body mass index (BMI) between 18 and $28 \mathrm{~kg} \mathrm{~m}^{-2}$ and an age between 18 and 50 years. Exclusion criteria included severe chronic disease (e.g. tumors, manifest coronary heart disease, DM1, DM2, severe kidney or liver conditions, endocrine and immunological conditions), gastrointestinal disorders (e.g. ulcers, chronic inflammatory bowel disease, celiac disease, pancreatitis) as well as status post gastrointestinal tract surgery (e.g. gastrectomy, short bowel syndrome, stomach reduction, gastric band, gastric balloon), lactose intolerance, pregnancy, competitive sports, lactation and alcohol or drug dependency. All subjects gave their written consent to participate in the study. Subjects were recommended not to donate blood for four weeks prior to the start of the intervention in order to avoid potential complications. In order to minimize fluctuations in fasting blood 
glucose and postprandial blood glucose response, the subjects were instructed not to consume any alcohol for three days prior to the study date, not to engage in a greater-than-usual level of physical activity and not to adhere to any weightreduction diets. In addition, each subject was provided with a standardized pasta dish as a last meal on the evening prior to each test day.

\subsection{Data analysis and statistics}

All data (age, height, weight, BMI, blood glucose, insulin) are given as mean value \pm standard deviation. For blood glucose and insulin, the incremental area under the curve (iAUC) values were calculated as per Brouns et al. 2005. ${ }^{16}$ Only the area under the curve through the fasting concentration was taken into account; the area below that was ignored. To calculate the iAUC, the raw data of the blood glucose and insulin concentrations were used and were determined for each subject as well as for both test and reference products. To calculate glucose and insulin concentrations at the various time points, the raw data were zero-value corrected. To calculate the iAUCs for blood glucose and insulin, a period of time from zero to two hours was taken into account. The GI of the cookies was then calculated from the iAUCs that were recorded for blood glucose. For this a ratio was created using the mean value of all individual iAUCs of the cookies (cookie) iAUC) and the reference iAUC. To calculate the iAUCs for insulin, a period of time from zero to two hours was likewise taken into account. As an additional parameter to compare the course of the blood glucose and insulin concentration, the $c_{\max }$ for the zero-value corrected values was calculated in each case. Statistical analysis was performed on the basis of the data's distribution type (Kolmogorov-Smirnov adjustment test). In the case of normal distribution, analysis was performed using single-factor analysis of variance (ANOVA); in the case of abnormally distributed data, the Mann-Whitney $U$ test was used. Statistical significance was determined to be $p<0.05$ and marked using an asterisk: ${ }^{*} p<0.05,{ }^{* *} p<0.005,{ }^{* *} p<0.001$. All statistical data analyses were performed with SPSS 19.0 (SPSS Inc., Chicago, Illinois, US).

\section{Results}

\subsection{Composition of test and reference products and starch- containing raw materials}

Table 1 shows the nutritional composition of the test and reference products. The total fiber content's in the fiber enriched cookies ( $6.5 \mathrm{~g}$ per $100 \mathrm{~g}$ ) and the digestive cookies ( $7.5 \mathrm{~g}$ per $100 \mathrm{~g}$ ) were more than three times as high as in white bread. The amount of polysaccharide components in the starch-containing raw materials of the fiber enriched cookies is summarized in Table 2 . The relatively high levels of glucans (3.91 g per $100 \mathrm{~g}$ DM) and pentosans (4.06 g per $100 \mathrm{~g} \mathrm{DM})$ both of them NSPS - in whole grain barley flour as well as the high glucan content ( $4.35 \mathrm{~g}$ per $100 \mathrm{~g} \mathrm{DM}$ ) in oat flakes are noteworthy here.

\subsection{Gelatinization characteristics of test and reference products and starch-containing raw materials}

When testing the gelatinization characteristics of the test and reference products, it was seen that white bread has the lowest transition enthalpy, while the fiber enriched cookie has the highest (Fig. 1A). The lower the transition enthalpy, the higher the degree of gelatinization. Thus the results indicate a high degree of starch gelatinization for white bread, while the degree of gelatinization is lowest for the fiber enriched cookie.

To determine the starches' gelatinization potential from the various starch-containing raw materials, the gelatinization enthalpy of the separated starch was considered in reference to the enthalpy of the relevant grain starches with "clean" surfaces (content of NSPS $<0.01 \mathrm{~g}$ per $100 \mathrm{~g}$ Starch from whole grain barley flour shows significantly limited gelatinization potential, while starch from soft white dunst has an optimal gelatinization capacity (Fig. 1B). The gelatinization potential of starch from oat flakes is moderate.

Light-microscopic investigation of starch based on partial gelatinization from the raw materials used provides information about the gelatinization state under conditions of water deprivation (Fig. 2A-E). Starch that is only slightly gelatinized appears dark. This can be seen especially well in starch

Table 1 Composition of the test and reference products

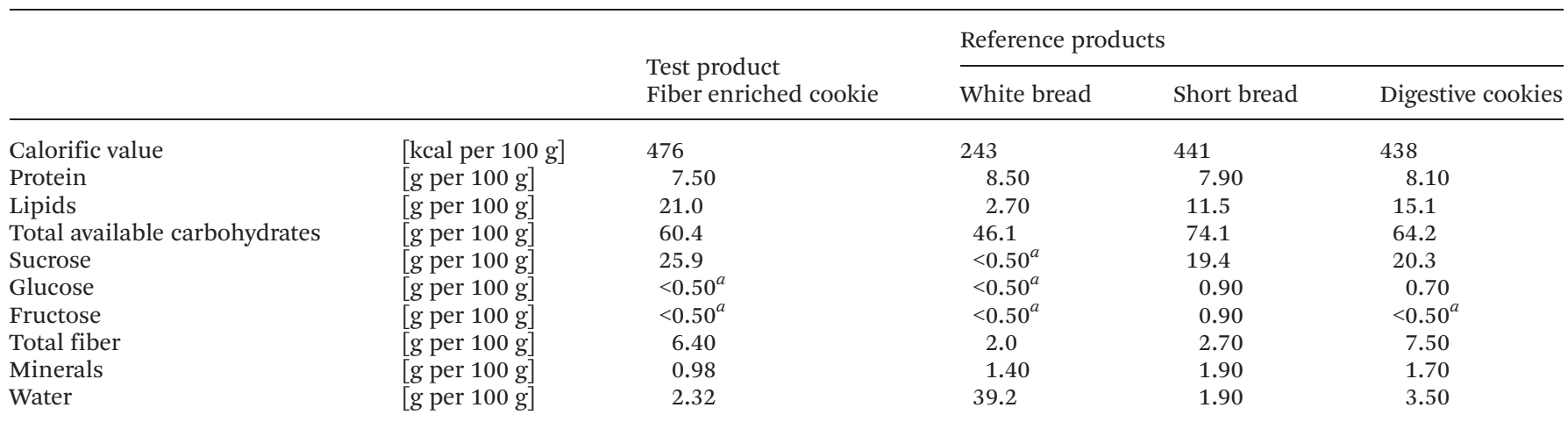

${ }^{a}$ Below detection limit. 
Table 2 Polysaccharide components in starchy raw materials

\begin{tabular}{|c|c|c|c|c|c|c|}
\hline & & $\begin{array}{l}\text { Whole grain } \\
\text { barley flour }\end{array}$ & Spelt flour & Oat flakes & $\begin{array}{l}\text { Soft wheat } \\
\text { dunst }\end{array}$ & $\begin{array}{l}\text { Whole grain } \\
\text { wheat flour }\end{array}$ \\
\hline Starch & [g per $100 \mathrm{~g}$ DM] & 65.9 & 69.1 & 59.7 & 71.9 & 63.6 \\
\hline Total pentosans & [g per $100 \mathrm{~g} \mathrm{DM}]$ & 4.06 & 1.25 & 1.82 & 0.49 & 2.38 \\
\hline
\end{tabular}

DM: dry matter.
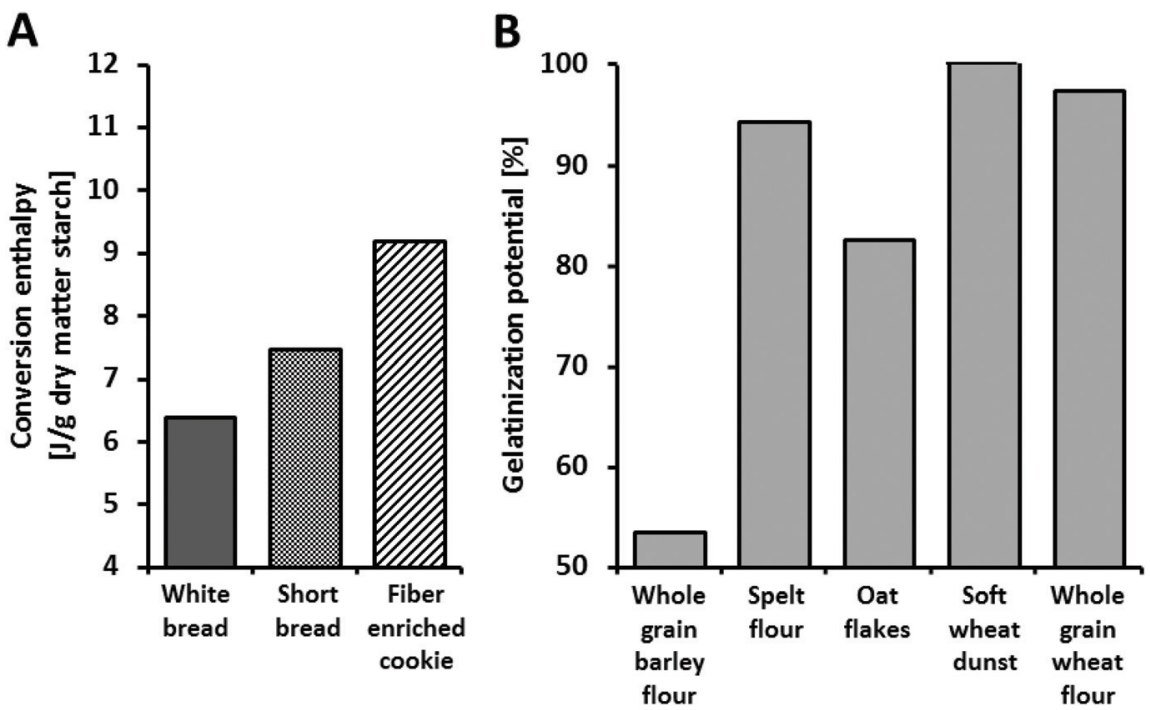

Fig. 1 Gelatinisation properties of the test and reference products and their starchy raw materials. (A) Conversion enthalpy of test product (fiber enriched cookie), reference products (white bread) and short bread. (B) Gelatinisation potential of starch separated from different starchy raw materials used in the test product.

from whole grain barley flour. The subsequently measured residual enthalpy for gelatinization of the starch granules at conditions of an excess of water (Fig. 2F) for whole grain barley flour was, at $0.85 \mathrm{~J}$ per $\mathrm{g}$ DM, very low compared with the other raw materials used.

\subsection{Characterization of separated starch granules}

The AFM tests on the topography of the starch granule surface area show that starch from oat flakes displays droplet-shaped deposits (Fig. 3B). The starch granule surface area of soft wheat dunst, on the other hand, has a primarily smooth surface (Fig. 3C). In starch from whole grain barley flour, there are portions of the surface area that have a smooth appearance and others that have irregular deposits (Fig. 3A). The adhesiveness measured, and assigned to the respective topography as a space-resolved map (in each case, images on the right in Fig. 3), show a distribution of adhesiveness that correlates with the composition of the surface areas of the individual starchcontaining raw materials. Evaluations of the surface adhesiveness in the form of histograms, in which the frequency (as a percentage) of specific adhesiveness of the starch granule surface area of various types of starch is compared (Fig. S1†), facilitate the results of the AFM tests on topography. The smooth appearance shown by soft wheat dunst starch in particular (Fig. 3C) is reflected in a homogeneous and broad distribution of adhesiveness (Fig. S1D†). The non-homogeneous adhesiveness distribution that oat starch displays (Fig. S1C $\dagger$ ) is consistent with the droplet-shaped deposits from Fig. 3B.

The results of quantitative analysis on the attachments to separated starch granules indicate the following: The starch granules separated out from whole grain barley flour have a high percentage of protein attachments; this is followed by oat flakes and spelt flour (Fig. 4A). Only whole grain barley flour displays a comparatively high level of glucan and pentosan attachment content. CLSM tests of the various types of starch with rhodamine $\mathrm{R}$ dye make the compact protein attachments visible (Fig. 4B-F). The three starch types from barley (Fig. 4B), spelt (Fig. 4C) and oats (Fig. 4D), have intensely red cluster type attachments which indicate that the starch granule surfaces are covered with protein complexes including NSPS. These complex attachments are, in contrast, less pronounced for soft wheat dunst (Fig. 4E) and whole grain wheat flour (Fig. 4F).

\subsection{In vitro digestion assays}

Differences between glucose concentrations of the three tested cookies and the reference product (white bread) after in vitro 

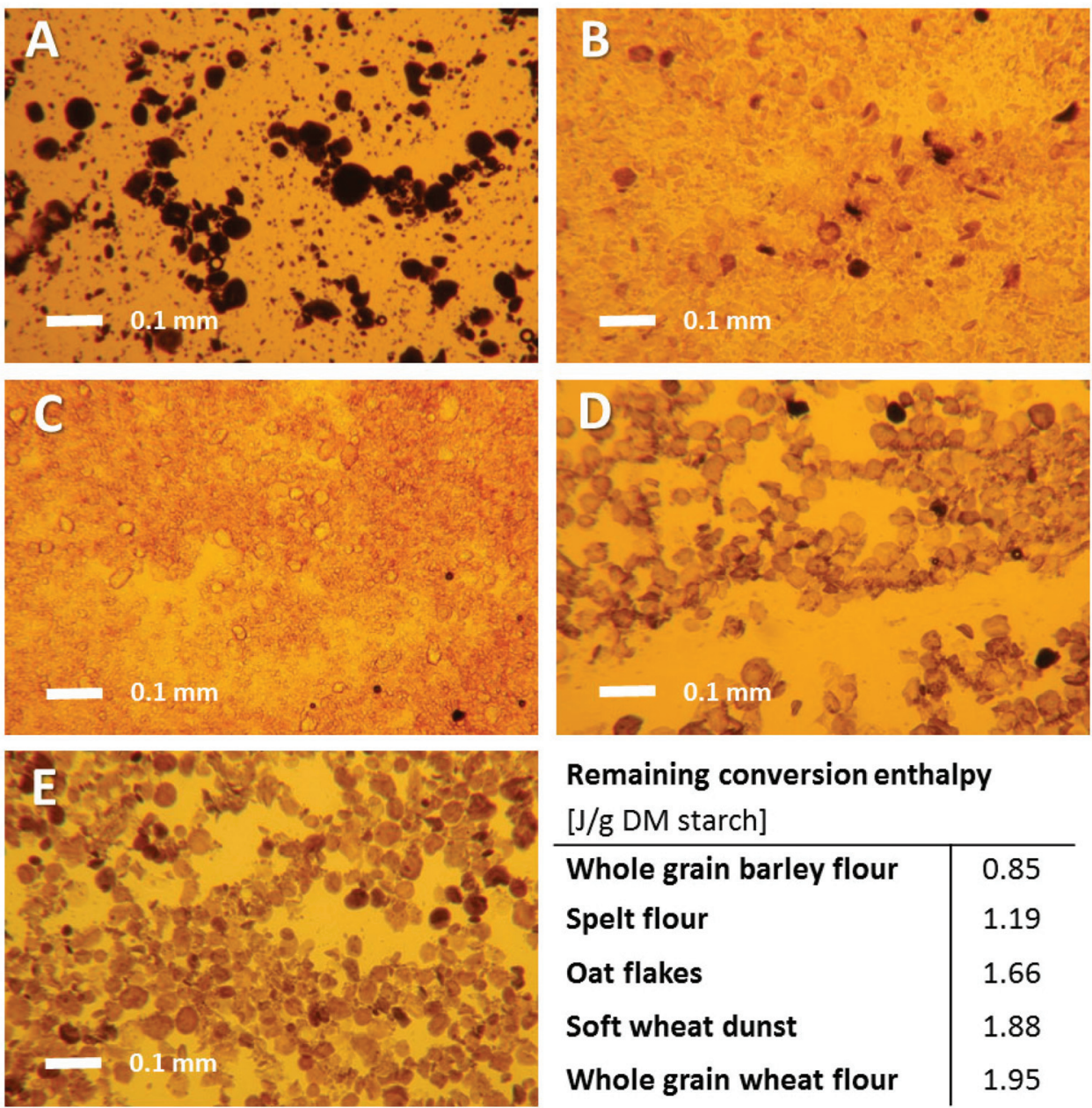

\section{Remaining conversion enthalpy}

[J/g DM starch]

\begin{tabular}{l|l}
\hline Whole grain barley flour & 0.85 \\
Spelt flour & 1.19 \\
Oat flakes & 1.66 \\
Soft wheat dunst & 1.88 \\
Whole grain wheat flour & 1.95
\end{tabular}

Fig. 2 Light microscopic pictured partly gelatinized starch from starchy raw materials of fiber enriched cookies. Dark areas: Ungelatinized starch granules; light areas: gelatinized starch after providing 40\% of water in relation to dry matter. Down right: Corresponding remaining conversion enthalpy in water surplus (A) whole grain barley flour; (B) spelt flour; (C) oat flakes; (D) soft wheat dunst; (E) whole grain wheat flour. DM: dry matter.

digestion assays were significant ( $p<0.001$ by ANOVA; Fig. 5). Short bread showed the highest absolute glucose release followed by digestive cookies. The lowest absolute glucose release was observed for the white bread followed by fiber enriched cookies. Considering the relative glucose release in \% of theoretical available glucose - which was calculated from the content of free glucose, sucrose and carbohydrates for each product - glucose concentrations after digestion of fiber enriched cookies were markedly lower at all time points compared to short bread and digestive cookies $(p<0.001$ by Sheffé post hoc tests).

\subsection{Nutritional physiological study and characteristics of the study population}

Table 3 gives an overview of the subjects' ages, BMIs and fasting glucose and insulin levels. The study collective consisted of 26 healthy men and women with a mean age of $28 \pm$ 7 years and a mean BMI of $24.1 \pm 2.5 \mathrm{~kg} \mathrm{~m}^{-2}$. The safety parameters that would indicate DM2 or impaired glucose tolerance (fasting glucose, fasting insulin and HbA1c) were within the reference range for all subjects and did not provide any signs of impaired glucose tolerance. No differences in the fasting concentrations of glucose and insulin were found between the two groups. All 26 subjects completed the nutritional physiological study. No cases of intolerance or gastrointestinal complaints were reported after consumption of the cookies.

\subsection{Glucose concentration in plasma and glycemic index}

The results for GI and other blood glucose and insulin metabolism parameters are summarized in Table 4 and Fig. 6 . The fiber enriched cookie shows markedly less effect on blood glucose concentration compared with the reference product. At 30,45 and 60 minutes after consumption of the fiber enriched cookie, blood glucose levels were significantly lower than after consumption of white bread ( $p<0.001$, Table 4, Fig. 6A). The $c_{\max }$ value of the test product was also significantly lower than after consumption of white bread ( $p<0.05$, Table 4$)$. The iAUC of the fiber enriched cookie was likewise significantly lower 

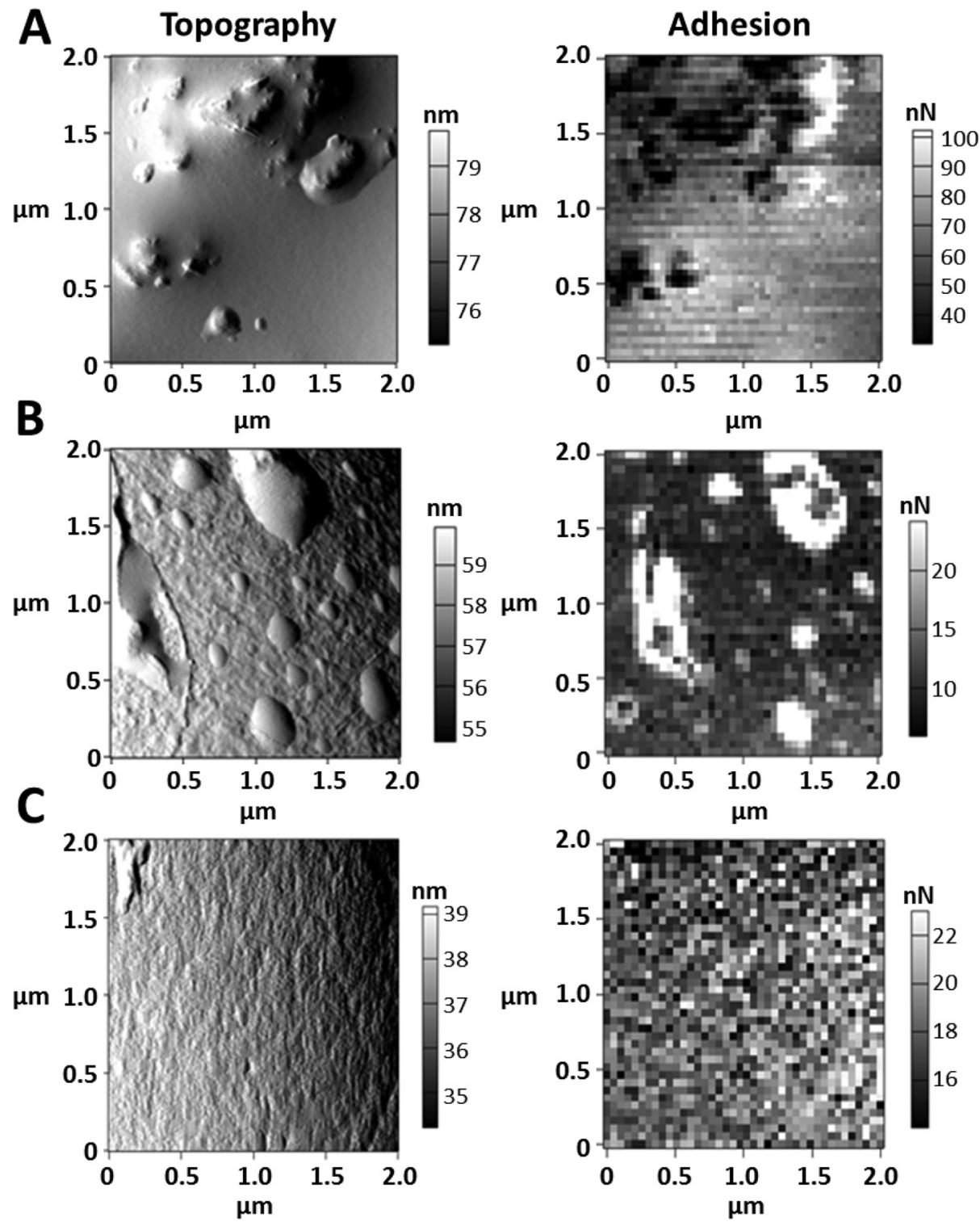

Fig. 3 Scanning electron microscopic characterization of starch granule surfaces from starchy raw materials of fiber enriched cookies. (A) Whole grain barley flour, (B) oat flakes and (C) soft wheat dunst. Topography (left): Elevations remarkable through dark and light changes. Drop-like bodies in A and B; smooth surface in C; partly smooth surface in A and B. Corresponding adhesion (right): high adhesive areas remarkable as light areas confirming surficial protein-NSPS complexes in A and B. nm: nano meter, $\mathrm{nN}$ : nano Newton.

compared with white bread ( $p<0.05$, Table 4 , Fig. 6B). The GI of the fiber enriched cookie was, at 59, significantly lower compared with white bread $(p<0.001)$ (Table 4 , Fig. 6C).

\subsection{Insulin concentration in plasma}

With white bread, the peak insulin concentration was reached after 45 minutes, while with the fiber enriched cookie, insulin levels had already begun to fall again after 30 minutes (Table 4, Fig. 6D). At 45, 60 and 90 minutes after consumption, insulin concentrations were significantly lower after consumption of the cookies compared with the reference product. The differences in the iAUC values for insulin between the fiber enriched cookie and the white bread (Table 4, Fig. 6E) were not significant, but they did display a trend $(p=0.071)$.

\section{Discussion}

With regard to GI, foods can be classified as having a low $(<55)$, moderate (55-69) or high GI $(>70)$. When measuring GI, glucose solution is generally used as reference. ${ }^{17}$ The GI of the fiber enriched cookie is 59 in relation to white bread as a reference. Using a conversion factor of 1.4 , this value can be converted in relation to a glucose solution as a reference. ${ }^{18}$ Accordingly, the fiber enriched cookie has a GI of 42 and 


\begin{tabular}{l|c|c|c|c|c}
$\mathbf{A}$ & $\begin{array}{c}\text { Whole } \\
\text { grain } \\
\text { barley } \\
\text { flour }\end{array}$ & $\begin{array}{c}\text { Spelt } \\
\text { flour }\end{array}$ & $\begin{array}{c}\text { Oat } \\
\text { flakes }\end{array}$ & $\begin{array}{c}\text { Soft } \\
\text { wheat } \\
\text { dunst }\end{array}$ & $\begin{array}{c}\text { Whole } \\
\text { grain } \\
\text { wheat } \\
\text { flour }\end{array}$ \\
\hline $\begin{array}{l}\text { Protein } \\
\text { [g/100g DM starch] }\end{array}$ & 8.67 & 2.20 & 4.53 & 0.39 & 0.98 \\
$\begin{array}{l}\text { Total glucans } \\
\text { [g/100g DM starch] }\end{array}$ & 0.64 & 0.11 & $<0.01$ & $<0.01$ & $<0.01$ \\
$\begin{array}{l}\text { Total pentosans } \\
\text { [g/100g DM starch] }\end{array}$ & 1.0 & 0.06 & 0.09 & 0.01 & 0.02
\end{tabular}
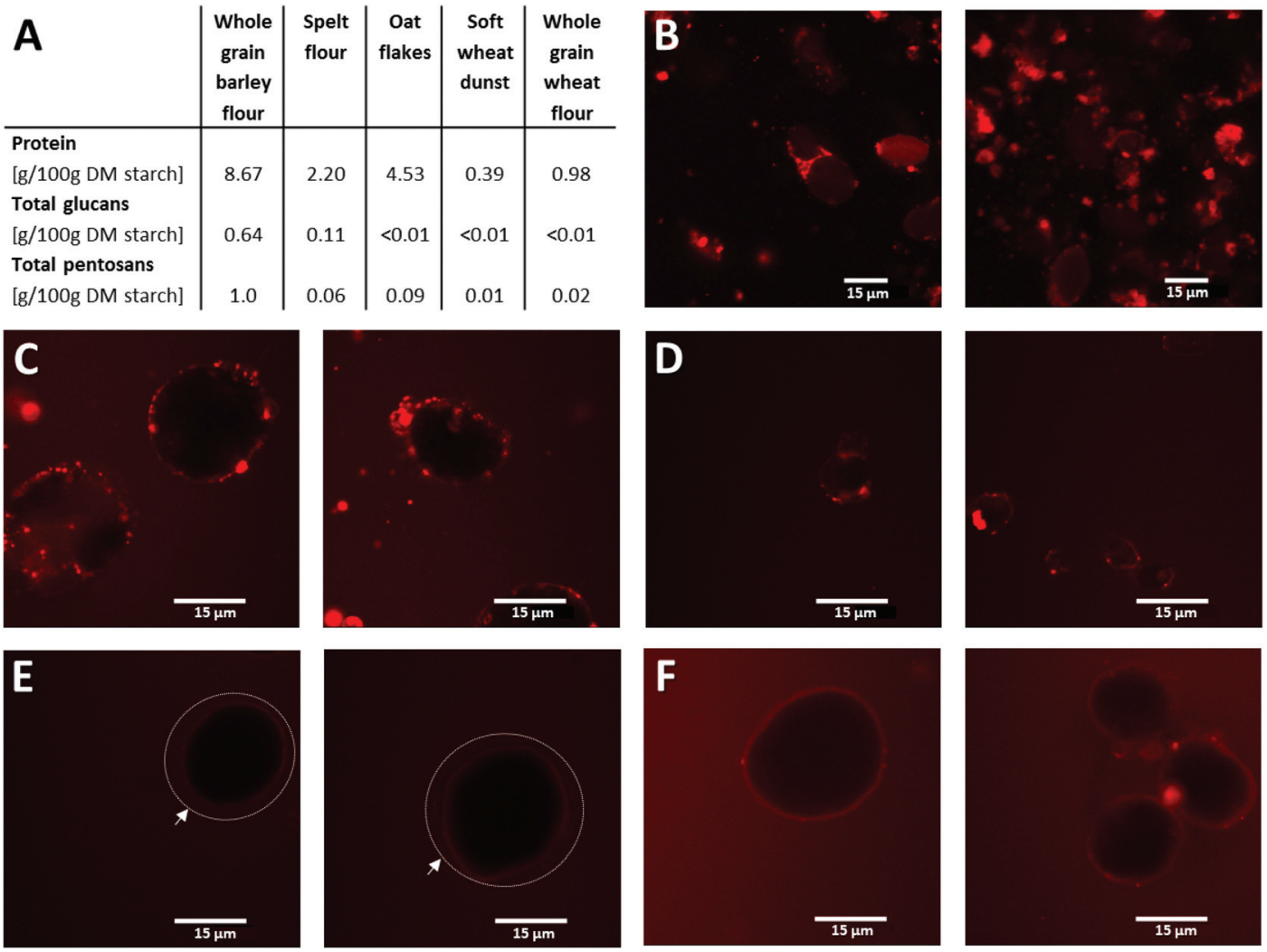

Fig. 4 Characterization of adherence on starch granules from starchy raw materials of fiber enriched cookies. (A) Amount of protein, total glucane and total pentosane adhering on granules of separated starch from starchy raw material. (B-F) Confocal laser scanning microscopic images of protein adherence on the surface of the starch granules. Adhesive protein-NSPS complexes on starch granule surface (stained with Rhodamin R) from (B) whole grain barley flour, (C) spelt flour, (D) oat flakes, (E) soft wheat dunst, and (F) whole grain wheat flour. Two images are shown in each case. DM: dry matter.

A

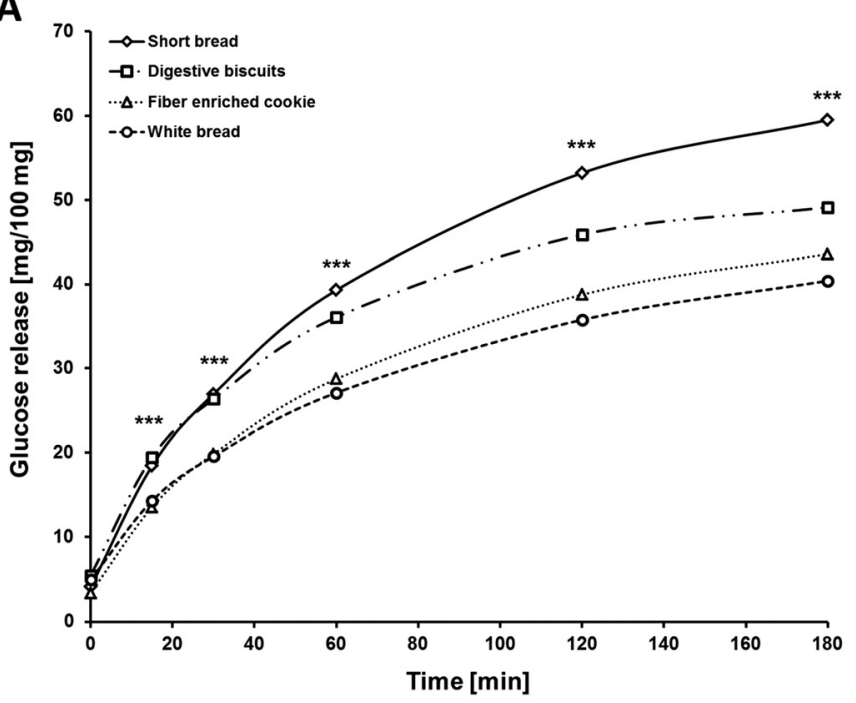

B

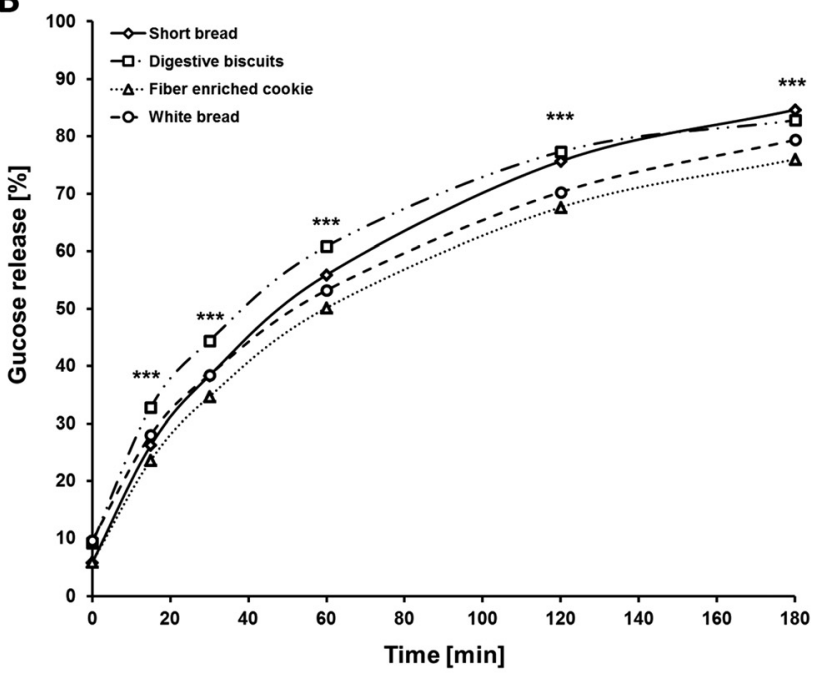

Fig. 5 Glucose release from the test product (fiber enriched cookie) and reference products (short bread, digestive cookies and white bread) after in vitro digestion. (A) Absolute glucose release in $\mathrm{mg}$ per $100 \mathrm{mg}$ product. (B) Relative glucose release in \% of theoretical available glucose which was calculated from the content of free glucose, sucrose and carbohydrates for each product. Cookies were digested in vitro using invertase, pancreatin and amyloglucosidase. Total free glucose was measured spectrometrically. Mean values of eightfold repeated experiments are shown. \# $p<0.001$ by ANOVA; $\ddagger p<0.001$ by Sheffé post hoc tests (fiber enriched cookie vs. short bread and digestive cookie), $\diamond p<0.005$ by Sheffé post hoc tests (fiber enriched cookie vs. white bread). 
Table 3 Characteristics of the study population

\begin{tabular}{lcl}
\hline & Mean $\pm \mathrm{SD}$ & Range \\
\hline Age [years] & $28 \pm 7$ & $19-43$ \\
BMI [kg m & \\
Fasting glucose $\left[\mathrm{mg} \mathrm{dl}^{-1}\right]$ & $24.1 \pm 2.5$ & $20.0-29.9$ \\
${\text { Fasting insulin }\left[\mu \mathrm{E} \mathrm{ml}^{-1}\right]}_{\mathrm{HbA}_{1 \mathrm{c}}[\%]}$ & $62.1 \pm 5.8$ & $71.0-96.0$ \\
& $4.9 \pm 0.3$ & $2.7-12.3$ \\
& & $4.2-5.3$
\end{tabular}

would thus be classified as a low-GI food. According to tests by Garsetti and co-workers ${ }^{19}$ the mean GI of various white flour cookies was $67 \pm 8$. In general, the GI of cookies is $51-57$ compared with glucose solution as a reference and is thus in the low to moderate GI range. ${ }^{18,20}$ For cookies with comparable ingredients (high amount of oat flakes and/or whole grains), a mean GI of $56 \pm 11$ compared with glucose solution was found. ${ }^{18}$ The GI of the fiber enriched cookie is thus markedly lower than the GI of those cookies.

The results of the physiological study with humans demonstrated that the polysaccharide digestibility of the fiber enriched cookies is impaired resulting in a delayed and reduced glucose and insulin increase in the blood. The in vitro data show that the glucose release is considerably lower from fiber enriched cookies compared to short bread ordinary digestive cookies and white bread (reference), which may explain the reduced blood glucose response.

The fiber content in the cookies tends to have a significant influence on blood glucose levels and GI. Primarily fiber-rich whole grain flours as well as oat fiber and oat flakes were used as ingredients for the cookies. In addition, the cookies were enriched with the water-soluble fiber NSPS inulin. The total fiber content of the cookies was $6.4 \mathrm{~g}$ per $100 \mathrm{~g}$. Soluble fiber like NSPS, by means of its gel-forming characteristics, slows starch digestion and glucose absorption and thereby prevents high postprandial glucose peaks. ${ }^{10,11,21-23}$ For example studies with $\beta$-glucan-enriched foods (oat bran breakfast cereals, bars, oat drinks, muffins) have shown a decrease in GI as well as lower maximum blood glucose in comparison to non-enriched foods. ${ }^{22-26}$ However, effects on blood glucose response were not observed until a total intake of $\geq 2.0 \mathrm{~g}$ of $\beta$-glucan was reached. ${ }^{25}$ The role of inulin in foods for the blood glucose response is contradictory. Various human studies have not observed any effects of inulin on glucose metabolism. ${ }^{27-29}$ In contrast, Marangoni \& Poli $(2008)^{30}$ observed a significantly lower GI of inulin enriched cookies ( $4.2 \mathrm{~g}$ inulin per $100 \mathrm{~g}$ and $6.0 \mathrm{~g}$ per $100 \mathrm{~g}$ total fiber) compared to control cookies without fiber enrichment. The possible influence of insoluble fiber on the digestion and absorption of carbohydrates and thus on postprandial glucose response is also controversial. ${ }^{31-34}$ Studies on this topic have come to opposing results. ${ }^{11,35}$

In addition, the microstructural and gelatinization characteristics of the starches from the starch-containing raw materials also potentially influence the carbohydrate digestion and postprandial glucose response of the fiber enriched cookies. We showed that the transition enthalpy of the fiber enriched cookies is high suggesting that enzymatic vulnerability of the starch is limited. One underlying cause is the high NSPS glucan content, which preferentially absorb water under conditions of water shortage, meaning starch gelatinization is prevented. As a result, accessibility of this partially gelatinized starch is difficult for pancreatic amylases and carbohydrate digestion and glucose absorption are thus less efficient. Analyses of the starch-rich raw materials show that whole grain barley flour and oat flakes in particular have a high NSPS content, which also explains their limited starch gelatinization potential. The lower the gelatinization potential under conditions of an excess of water, the more the surface of the starch granules is protected against penetration by water and thereby decrease starch gelatinization. The starch granules of the starch phase separated out from whole grain barley

Table 4 Glycemic index, adjusted glucose and insulin concentration after consumption of the test product (fiber enriched cookie) and reference product (white bread) $(n=26)$

\begin{tabular}{|c|c|c|}
\hline Parameter & $\begin{array}{l}\text { White bread } \\
\text { Mean } \pm \text { SD }\end{array}$ & $\begin{array}{l}\text { Fiber enriched } \\
\text { cookie } \\
\text { Mean } \pm \text { SD }\end{array}$ \\
\hline Glycemic index & $100 \pm 0$ & $58.9 \pm 29.7^{* * *}$ \\
\hline iAUC $_{0-2} \mathrm{~h}$ glucose $\left[\mathrm{mg} \mathrm{dl}^{-1} \mathrm{~min}^{-1}\right]$ & $2348 \pm 1370$ & $1332 \pm 892^{* *}$ \\
\hline Glucose $c_{\max }\left[\mathrm{mg} \mathrm{dl}^{-1}\right]$ & $42.6 \pm 15.2$ & $27.8 \pm 12.0^{* * *}$ \\
\hline Glucose conc. $30 \mathrm{~min}\left[\mathrm{mg} \mathrm{dl}{ }^{-1}\right]$ & $37.9 \pm 13.9$ & $24.5 \pm 12.7^{* * *}$ \\
\hline Glucose conc. $45 \mathrm{~min}\left[\mathrm{mg} \mathrm{dl}{ }^{-1}\right]$ & $32.1 \pm 20.0$ & $12.7 \pm 19.6^{* * *}$ \\
\hline Glucose conc. $60 \mathrm{~min}\left[\mathrm{mg} \mathrm{dl}{ }^{-1}\right]$ & $18.1 \pm 22.0$ & $2.8 \pm 19.1^{* * *}$ \\
\hline Glucose conc. $90 \mathrm{~min}\left[\mathrm{mg} \mathrm{dl}^{-1}\right]$ & $4.7 \pm 18.6$ & $0.8 \pm 15.3$ \\
\hline iAUC $_{0-2} \mathrm{~h}$ insulin $\left[\mu \mathrm{E} \mathrm{ml}^{-1} \mathrm{~min}^{-1}\right]$ & $4013 \pm 2172$ & $3063 \pm 1484$ \\
\hline Insulin $c_{\max }\left[\mu \mathrm{E} \mathrm{ml}^{-1}\right]$ & $48.9 \pm 19.4$ & $41.6 \pm 16.4^{*}$ \\
\hline Insulin conc. $30 \mathrm{~min}\left[\mu \mathrm{E} \mathrm{ml}{ }^{-1}\right]$ & $36.6 \pm 18.7$ & $34.9 \pm 17.9$ \\
\hline Insulin conc. $45 \mathrm{~min}\left[\mu \mathrm{E} \mathrm{ml} l^{-1}\right]$ & $43.1 \pm 17.0$ & $33.5 \pm 18.3^{*}$ \\
\hline Insulin conc. $60 \mathrm{~min}\left[\mu \mathrm{E} \mathrm{ml}^{-1}\right]$ & $37.4 \pm 17.7$ & $25.8 \pm 15.6^{* * *}$ \\
\hline Insulin conc. $90 \mathrm{~min}\left[\mu \mathrm{E} \mathrm{ml}{ }^{-1}\right]$ & $27.9 \pm 20.1$ & $17.2 \pm 12.8^{* *}$ \\
\hline
\end{tabular}

${ }^{*} p<0.05,{ }^{* *} p<0.005,{ }^{* *} p<0.001$ in comparison with white bread. 

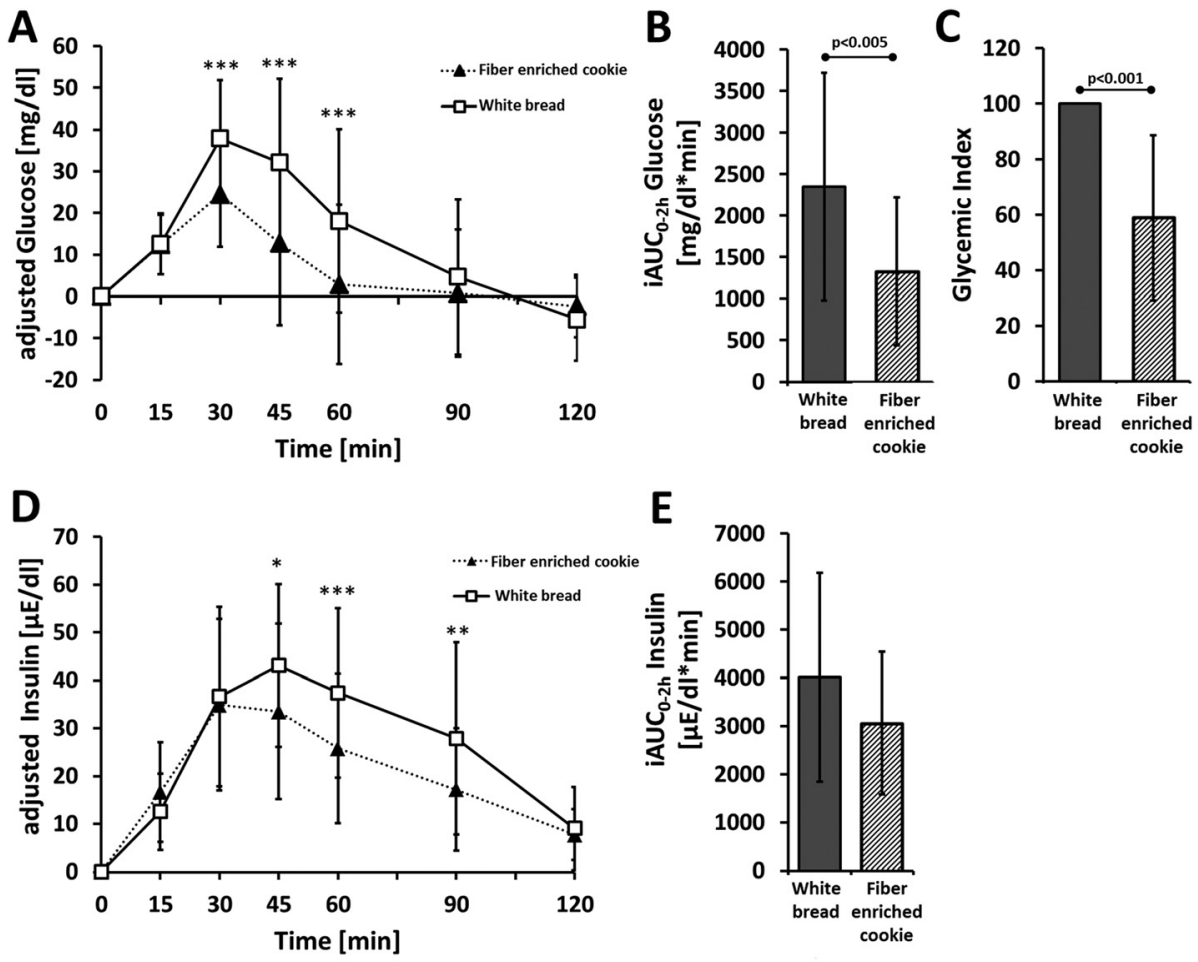

Fig. 6 Glucose and insulin parameters after consumption of the test product (fiber enriched cookie) and reference product (white bread) ( $n=26$ ). (A) Glycemic index, (B) adjusted glucose course, (C) incremental area under curve (iAUC) for glucose concentration, (D) adjusted insulin course and (E) incremental area under curve (iAUC) for insulin concentration. ${ }^{*} p<0.05,{ }^{*} p<0.005,{ }^{* *} p<0.001$

flour and oat flakes display the highest percentages of surface attachments in the form of proteins as well as glucans and pentosans provoking poor enzymatic vulnerability. The low residual enthalpy that was measured for the starch from whole grain barley flour can be explained by limited accessibility or sealing off of the starch granule surface due to a shortage of water. This means that the starch granules of whole grain barley flour can no longer gelatinize even when there is an excess of water. The starch granule surface area of whole grain barley flour, spelt flour and oat flakes bear cluster-shaped proteinNSPS complexes, which confirm the results of atomic/scanning force microscopy analyses of the topography and adhesiveness of the starch granules. The adhesiveness distribution and the surface area topography of the starch granules are thus significantly influenced by the attached protein-NSPS complexes.

\subsection{Strength and limitations}

One weakness of the nutritional physiological study is the absence of a low-fiber cookie and other digestive cookie for comparison, which would likewise alleviate comparability between in vivo and in vitro data. Although the expected difference in GI between white bread and the cookies can indeed be demonstrated, these foods differ markedly in their nutritional composition. One strength of this study is its straight forward methodological design and close adherence to the methodological recommendations for GI measurement. ${ }^{12,16}$ Inter-individual fluctuations could thus be prevented as far as possible.

\section{Conclusion}

In summary, this study shows that the fiber enriched cookie is a low-GI food. The low GI of the fiber enriched cookies can be attributed to the high fiber content, especially soluble fiber like glucans and inulin, as well as to the microstructural and gelatinization characteristics of the starches in the starch-containing raw materials that were used. The starch granules from whole grain barley flour and oat flakes in particular show a high percentage of surface attachments in the form of proteinNSPS complexes, which potentially impede starch gelatinization and thus enzymatic vulnerability. Fiber enriched cookies thus offer an alternative to other carbohydrate-rich snacks to induce lower blood glucose and insulin response.

\section{Abbreviations}

AFM Atomic force microscope

ANOVA Analysis of variance

BMI Body mass index

CLSM Confocal laser scanning microscopy

DM Dry matter

DM2 Diabetes mellitus type 2

DSC Differential scanning calorimetry

FAO Food and agriculture organization of the United Nations 
GCP Good clinical practice

GI Glycemic index

iAUC Incremental area under the curve

NSPS Non-starch polysaccharides

\section{Acknowledgements}

The provision of the test products by Bahlsen GmbH \& Co. KG (Hannover, Germany) is kindly acknowledged. The authors are solely responsible for the design and conduct of the study, collection, management, analysis, and interpretation of the data, as well as preparation of the manuscript. All authors had full access to the data and take responsibility for its integrity. All authors have read and agree with the manuscript as written. We would like to thank the participants who contributed their time to this project.

\section{References}

1 A. W. Barclay, P. Petocz, J. McMillan-Price, V. M. Flood, T. Prvan, P. Mitchell and J. C. Brand-Miller, Am. J. Clin. Nutr., 2008, 87, 627-637.

2 J. Salmerón, J. E. Manson, M. J. Stampfer, G. A. Colditz, A. L. Wing and W. C. Willett, Diabetes Care, 1997, 20, 545550.

3 X.-y. Ma, J.-p. Liu and Z.-y. Song, Atherosclerosis, 2012, 223, 491-496.

4 Y. Choi, E. Giovannucci and J. E. Lee, Br. J. Nutr., 2012, 108, 1934-1947.

5 G. Livesey, R. Taylor, T. Hulshof and J. Howlett, Am. J. Clin. Nutr., 2008, 87, 258S-268S.

6 Anonymous, Eur. J. Clin. Nutr., 2000, 54, 353-355.

7 H. Connor, F. Annan, E. Bunn, G. Frost, N. McGough, T. Sarwar and B. Thomas, Diabetes Med., 2003, 20, 786-807.

8 R. D. DiPaola, R. Asis and M. A. J. Aldao, Starch/Stärke, 2003, 55, 403-409.

9 S. Tamaki, K. Teranishi, M. Hisamatsu and T. Yamada, Starch/Stärke, 1997, 49, 387-390.

10 A. L. Jenkins, D. J. Jenkins, T. M. Wolever, A. L. Rogovik, E. Jovanovski, V. Božikov and V. Vuksan, Croat. Med. J., 2008, 49, 772-782.

11 D. J. Jenkins, T. M. Wolever, A. R. Leeds, M. A. Gassull, P. Haisman, J. Dilawari, D. V. Goff, G. L. Metz and K. G. Alberti, Br. Med. J., 1978, 1392-1394.

12 Joint FAO/WHO Expert Consultation on Carbohydrates in Human Nutrition, Carbohydrates in human nutrition, World Health Organization, Food and Agriculture Organization of the United Nations, Rome, 1998.
13 B. V. McCleary and R. Codd, J. Sci. Food Agric., 1991, 55, 303-312.

14 A. M. Kiszonas, C. M. Courtin and C. F. Morris, Cereal Chem., 2012, 89, 143-150.

15 H. J. Butt, B. Cappella and M. Kappl, Surf. Sci. Rep., 2005, 59.

16 F. Brouns, I. Bjorck, K. N. Frayn, A. L. Gibbs, V. Lang, G. Slama and T. M. S. Wolever, Nutr. Res. Rev., 2005, 18, 145.

17 B. J. Venn and T. J. Green, Eur. J. Clin. Nutr., 2007, 61, S.122-S.131.

18 K. Foster-Powell, S. H. Holt and J. C. Brand-Miller, Am. J. Clin. Nutr., 2002, 76, 5-56.

19 M. Garsetti, S. Vinoy, V. Lang, S. Holt, S. Loyer and J. C. Brand-Miller, J. Am. Coll. Nutr., 2005, 24, 441-447.

20 K. N. Englyst, S. Vinoy, H. N. Englyst and V. Lang, Br. J. Nutr., 2003, 89, 329.

21 J. W. Anderson, D. B. Spencer, C. C. Hamilton, S. F. Smith, J. Tietyen, C. A. Bryant and P. Oeltgen, Am. J. Clin. Nutr., 1990, 52, 495-499.

22 K. M. Behall, Diabetes Care, 2006, 29, 976-981.

23 A. L. Jenkins, D. J. Jenkins, U. Zdravkovic, P. Würsch and V. Vuksan, Eur. J. Clin. Nutr., 2002, 56, 622-628.

24 H. Kim, K. S. Stote, K. M. Behall, K. Spears, B. Vinyard and J. M. Conway, Eur. J. Nutr., 2009, 48, 170-175.

25 H. Mäkeläinen, H. Anttila, J. Sihvonen, R.-M. Hietanen, R. Tahvonen, E. Salminen, M. Mikola and T. SontagStrohm, Eur. J. Clin. Nutr., 2007, 61, 779-785.

26 N. Tapola, H. Karvonen, L. Niskanen, M. Mikola and E. Sarkkinen, Nutr., Metab. Cardiovasc. Dis., 2005, 15, 255261.

27 J. Causey, J. M. Feirtag, D. D. Gallaher, B. C. Tungland and J. L. Slavin, Nutr. Res., 2000, 20, 191-201.

28 R. Giacco, G. Clemente, D. Luongo, G. Lasorella, I. Fiume, F. Brouns, F. Bornet, L. Patti, P. Cipriano, A. A. Rivellese and G. Riccardi, Clin. Nutr., 2004, 23, 331-340.

29 D. Letexier, F. Diraison and M. Beylot, Am. J. Clin. Nutr., 2003, 77, 559-564.

30 F. Marangoni and A. Poli, Nutr., Metab. Cardiovasc. Dis., 2008, 18, 602-605.

31 C. W. C. Kendall, A. Esfahani and D. J. Jenkins, Food Hydrocolloids, 2010, 24, 42-48.

32 J. M. Lattimer and M. D. Haub, Nutrients, 2010, 2, 12661289.

33 D. Mudgil and S. Barak, Int. J. Biol. Macromol., 2013, 61, 16.

34 K. Raninen, J. Lappi, H. Mykkänen and K. Poutanen, Nutr. Rev., 2011, 69, 9-21.

35 T. M. S. Wolever, Am. J. Clin. Nutr., 1990, 51, 72-75. 